\title{
Prospectiva e innovación tecnológica
}

\author{
Prospective and technological innovation
}

\author{
Sandy Villa Ruvalacaba ${ }^{a}$, Judith Alejandra Velázquez Castro ${ }^{b}$, Erika Cruz Coria $^{c}$
}

\begin{abstract}
:
Innovation is a fundamental for a constant growth for any company. This must be kept constant in organizations, either in administration area or production, with the purpose of optimize resources and achieve to establish competitive advantages. In this sense, this book takes up the subject of technological foresight and innovation, which makes a company competitive due to its changes that arise in a globalized world. Therefore, technology and innovation must take part of the duties that every manager must do: first is to acquire, develop and allocate resources and; the second is the development and exploitation of the organization's capabilities for innovation. Therefore, the book is composed of eight valuable chapters to provide an overview of the subject.
\end{abstract}

Keywords:

Innovation, investigation, competitive advantage, technologic advances

\section{Resumen:}

La innovación es fundamental para el crecimiento constante de cualquier empresa. Ésta debe mantenerse constante en las organizaciones, ya sea en el área de administración o en la de producción, con el fin de optimizar recursos y lograr establecer ventajas competitivas. En ese sentido, el presente libro retoma el tema de prospectiva tecnológica e innovación, que hacen que una empresa sea competitiva debido a que estas dos ramas representan una base sólida para estar a la vanguardia de los cambios que se suscitan en un mundo globalizado. Así, la tecnología y la innovación deben formar parte de las dos tareas que debe llevar a cabo cualquier administrador: la primera es adquirir, desarrollar y asignar recursos y; la segunda es el desarrollo y explotación de las capacidades de la organización para la innovación. Por lo anterior, para proporcionar un panorama general del tema, el libro se constituye de ocho valiosos capítulos.

\section{Palabras Clave:}

Innovación, investigación, ventaja competitiva, avances tecnológicos

\section{Prospectiva e innovación tecnológica}

El primer capítulo: Consideraciones básicas en la gestión de la tecnología; discurre sobre la competitividad en una organización, e indica que esta depende de la eficiencia con el que se administren los recursos tecnológicos, materiales y los financieros, como también de la eficiencia en la producción o procesos. En éste, se resalta que la calidad, la velocidad y flexibilidad en la toma decisiones y el grado de adaptación a cambios en el entorno, son elementos estratégicos importantes en la realización de la competitividad, la cual entrega el valor otorgado al cliente.
Por otra parte, Ferraro y Lerch (1997) resaltan una forma de clasificar las tecnologías: como blandas y duras; mencionan que estas son aplicables en cualquier institución u organización para el cumplimiento de sus objetivos, y abarcan empresas industriales, comerciales, de servicios o instituciones con o sin fines de lucro. Otra clasificación de tecnología es: 1) tecnología hardware, incorporada en las maquinas, (puede ser tangible); 2) tecnología software, que se presenta a través de impresos como manuales, libros o revistas científicos, así como en programas de computadoras; 3) tecnología orgware, está reflejada en las estructuras organizacionales; y 4) tecnología humanware, incorporada a las personas, quienes tienen un know how. Así se observa que la

\footnotetext{
${ }^{a}$ Estudiante de la Licenciatura en Turismo, Instituto de Ciencias Económico Administrativas de la Universidad Autónoma del Estado de Hidalgo, https://orcid.org/0000-0002-8433-6278, villasandy5@gmail.com

${ }^{\mathrm{b}}$ Autor de Correspondencia, Doctora en Estudios Turísticos, Área Académica de Turismo, Instituto de Ciencias Económico Administrativas de la Universidad Autónoma del Estado de Hidalgo, https://orcid.org/0000-0001-7138-9293, judithalejandra666@ gmail.com

'Doctora en Ciencias Ambientales, Área Académica de Turismo, Instituto de Ciencias Económico Administrativas de la Universidad Autónoma del Estado de Hidalgo, https://orcid.org/0000-0001-7984-0069, ecoria84@ hotmail.com
} 
innovación va de la mano con la manifestación de tecnologías.

Además, la OCDE y Eurostat (2005) mencionan que el surgimiento de la innovación se lleva a través de nuevas ideas o partir del mejoramiento de ideas ya existentes. Para el logro de esto, es necesario apoyarse de la investigación y generar innovaciones. En ese sentido, se distinguen cuatro tipos de innovación: 1) de producto, 2) de proceso, 3) de mercadotecnia y de 4) de organización.

El capítulo dos: Gestión estratégica y evaluación de innovación, se divide en cinco partes. La primera habla sobre los modelos estratégicos. En la segunda parte presenta el modelo de gestión estratégica balanced scorecard. La tercera parte se analiza la aplicación de este. En la cuarta parte se presenta una aproximación de este y en la quinta parte se presenta la conclusión.

Con el paso a la globalización y apertura comercial que esta desenlaza, obliga a todas las organizaciones a estar en constante actualización de tecnologías e innovaciones, ya que, si no es así, se verían estancados junto con la competencia poco competente. La apertura a la globalización conlleva a ampliar el mercado, pero, así como se perciben nuevas oportunidades se obtendrá mayor competencia, ya sea nacional o internacional con mejor especializada o en vías a la especialización. Por ello, se afirma la importancia de la creación de ventajas competitivas. Como cualquier actividad, antes de ser optada a ser aplicada, esta debe de ser evaluada, lo mismo sucede para la innovación.

Así, en el capítulo segundo se explica la metodología de la gestión y de evaluación de la innovación. La gestión estratégica se encuentra en constantes evoluciones debido a los avances de las tecnologías, lo que conlleva a que se vayan creando más y más necesidades a satisfacer, tanto de forma exterior como interna para el éxito de una organización. Por ende, toma importancia el crear estrategias competitivas adecuadamente planeadas e implementadas.

Porter, (1982) diseño el diagrama para el desarrollo de una estrategia competitiva. Donde toma los factores internos de la empresa como las fuerzas y debilidades de la empresa y los valores personales de los ejecutivos clave y los factores externos de la empresa, que son oportunidades y riesgos del sector industrial y expectativas sociales de más amplitud. Porter correlaciona todos los factores entre ellos para analizarlos y tomarlos en cuenta a la hora de crear la estrategia competitiva y así lograr la diferencia en el mercado. Esta ventaja busca crear valor añadido para los clientes, accionistas y el personal que labora en la organización.

Estos valores son importantes para la creación del modelo de gestión estratégica balanced scorecard (BSC). EI BSC, surge tras el objetivo de que las organizaciones sean exitosas, invirtiendo en el capital intelectual y gestionándolo correctamente. Sustituyendo los procesos estandarizados por innovadores, flexibles con alta calidad. Dando autorización al personal a innovar y mejorar los productos, servicios y procesos. El BSC, simplifica la toma de decisiones, y la valoración y medición del seguimiento de las actividades o procesos que generan valor al personal, clientes, proveedores y actuales o futuros accionistas.

El elemento central del BSC se enfoca en las finanzas, ya que de aquí parten las decisiones y planes estratégicos para el logro de los objetivos planteados. A lo que conlleva a resaltar las ventajas de utilizar el BCS, facilita el gestión y control, gracias a su reducido número de indicadores, cooperación y relación con todos los niveles de la organización, facilitación de observar las causas y efectos, facilitando el entendimiento de los objetivos estratégicos para su implementación.

Como cualquier método, este tiene sus deficiencias, por ende, se apoya con la inclusión de la dinámica de sistemas, lo que le apoyara a integrar las retroalimentaciones y relaciones no lineales. Mejorará los flujos y estoques, así como detectar los efectos en ellos por los retrasos. Mejorará el flujo de información entre niveles diferentes. Distinción entre factores de control y largo plazo. Elección de la escala adecuada para el análisis de la retroalimentación. EI BSC, por ser dinámico con las cuatro perspectivas mencionadas anteriormente facilita la innovación en estas.

En la perspectiva financiera requiere de estabilidad en corto plazo y autocontinuidad de la organización. En la perspectiva del cliente se buscarán nuevas formas de satisfacerlo. En la perspectiva del aprendizaje y crecimiento se buscarán los procedimientos para crear valor de los intangibles. En la perspectiva de procesos internos se buscarán los procesos o productos que añadan valor tanto al cliente como a la empresa. En los procesos internos es donde se deberán desarrollar las innovaciones.

La gestión estratégica toma en cuenta el corto y largo plazo, enfatiza las respuestas estratégicas ante escenarios imprevistos. Apoya en la previsión de eventos para poder determinar los impactos posibles de decisiones con efectos en el futuro. El uso del BSC en esta actividad de gestión estratégica resulta de gran apoyo a 
diferentes campos de la organización. Ayuda en la actividad organizacional, finanzas, satisfacción del cliente, procesos internos y en las actividades de innovación.

El capítulo tres, versa sobre la importancia de las tecnologías para obtener ventajas competitivas en los negocios, en este se explican las tres técnicas de producción famosas como, taylorismo, fordismo y toyotismo. El taylorismo dio iniciativa a la plusvalía, en el fordismo fue el pionero en utilizar el just in time en sus procesos. El toyotismo a parte de mejorar las debilidades del fordismo, se basó en la flexibilidad laboral, y fomento el trabajo en equipo.

Además, retoma el termino empresario schumpeteriano, que es utilizado para referirse a los empresarios innovadores, que gracias a su creatividad, capacidad organizativa y mejoras de eficiencia han podido crecer empresarialmente más aplicando o llegando a desarrollar tecnologías nuevas.

En el capítulo cuarto, fundamentos de la prospección tecnológica y casos, se mencionan algunos ejemplos de métodos de estudios en casos aplicados. Como bien se sabe, el estudio es el inicio importante para cada proyecto y se inicia haciendo una investigación. La investigación debe ser de manera interna y externa para mayor estudio de campo.

Para poder obtener una ventaja estratégica es igual a la prospectiva tecnológica, con el estudio adecuado dará la herramienta para la correcta toma de decisión. La prospectiva tecnológica se obtiene después haber analizado y obtenido datos del estudio de investigación. El PT (prospectiva tecnológica) puede darse en ocasiones la tarea de determinar el escenario tecnológico posible en unos años, y en función de ello adoptar decisiones.

En el capítulo cinco, se asegura que el uso de estadísticas como método de prospección tecnológica es de gran importancia, debido a que con las estadísticas puede realizar un análisis cuantitativo. Las áreas que abarca las estadísticas son vastas, no solo se encarga de agrupar datos y mostrarlos en gráficas, las estadísticas ayudan a realizas una prospección. Las estadísticas abarcan la agrupación y presentación de datos, análisis de regresión, series de tiempo, medidas de tendencia central y dispersión, probabilidades, muestreo, estimación, pruebas de hipótesis, control de calidad y teoría de decisiones.

Por ello, la estadística se divide en dos grupos, la estadística descriptiva estadística inferencial. La estadística descriptiva es la que se dedica al ordenamiento de la información para la presentación por medio de tablas o graficas de igual forma como en la obtención de parámetros para explicar dicha información. La estadística inferencial se refiere a los métodos utilizados para hacer conclusiones. Debido a los pasos continuos a las mejoras e implementaciones tecnológicas, las empresas están casi obligadas a la actualización e implementación de la tecnología. De acuerdo con la OCDE, en México existen muy pacas empresas cuentan con un centro de desarrollo tecnológico.

En el capítulo seis, intitulado: Caso de prospección tecnológica: la industria aeronáutica mexicana, se establece que en estudios presentados por Mexico Now, se muestra a México como el país que ha tomado ventaja sobre los otros países de principal competencia, como: India, China y Brasil. Por lo que, México es ahora capaz de ofrecer un ahorro en costos generales de manufactura de 22 a $25 \%$, en comparación con los Estados Unidos.

Los principales países que invierten en la industria aeroespacial en México son Estados Unidos, Canadá, Francia, España y Reino Unido; The Everest Group (2008). La capacidad mexicana en el sector aeronáutico son manufactura, ingeniería y servicio MRO. El estado con mayor ventaja para dicho sector es el estado de Querétaro debido a su alto contenido de innovación.

El capítulo siete versa sobre la radiofrecuencia para identificación aplicada a la gestión de recursos de negocio, en el que se explica la importancia de la optimización de inventarios y el control de los mismos, para una organización. Ya sea para inventarios de material prima, en procesos y de productos terminados. Así, los sistemas de gestión por chips de radiofrecuencia (RFID) se define como un sistema de almacenamiento y recuperación de datos remoto que usa dispositivos denominados etiquetas, transpondedor o tags RFID. Estos sistemas pueden clasificarse de acuerdo a la frecuencia de radio que usan: 1) frecuencia baja; 2) frecuencia alta; 3) frecuencia ultra alta; y 4) frecuencia de microondas.

Ahora, el concepto de calidad se ve envuelto en una cadena de suministros, y se puede hablar de que una empresa está ligada a sus proveedores; la empresa inicial es proveedora de otra y así sucesivamente, determinando una cadena que termina en el usuario o cliente final.

Por último, en el capítulo ocho, se aborda la prospección tecnológica, la cual no es un término de simple moda, es la herramienta que ayudara a proyectar posibles escenarios a contemplar. Como condición de éxito en el método, este exigirá un análisis posterior, la discusión de dicho análisis, la difusión a la que se dedicará dicho 
esfuerzo, tiempo y recursos. Esto con el objetivo que se desarrollen estrategias pertinentes, brindado una guía eficaz para la toma de decisiones con fundamentos y disminuir la incertidumbre que produzca las decisiones estratégicas.

\section{Referencias}

[1] Gómez, Denisse (2011). Prospectiva e innovación tecnológica. Ed. XXI, México, 205 p. 